\title{
Modelagem Analítica da Geração de Bandas de Deformação em Reservatórios Areníticos
}

Leandro Guedes Carvalho ${ }^{\star 1,2}$, Anderson Moraes ${ }^{2}$, Raquel Quadros Velloso ${ }^{1}$, Jorge André Braz de Souza ${ }^{2}$, Jorge Campos Junior ${ }^{2},{ }^{1}$ Pontifícia Universidade Católica do Rio de Janeiro, ${ }^{2}$ Petróleo Brasileiro S.A.

\section{Copyright 2021, SBGf - Sociedade Brasileira de Geofísica}

This paper was prepared for presentation during the $17^{\text {th }}$ International Congress of the Brazilian Geophysical Society held in Rio de Janeiro, Brazil, 16-19 August 2021.

Contents of this paper were reviewed by the Technical Committee of the $17^{\text {th }}$ International Congress of the Brazilian Geophysical Society and do not necessarily represent any position of the SBGf, its officers or members. Electronic reproduction or storage of any part of this paper for commercial purposes without the written consent of the Brazilian Geophysical Society is prohibited.

\section{Resumo}

The permeability prediction is a critical step in the flow of geological modeling and reservoir characterization and is essential for the development of production projects. Subseismic structures called deformation bands (BD) can reduce permeability up to six orders of magnitude in relation to the host reservoir sandstone and can thus act as total or partial barriers to the fluid flow.

The present work intends to contribute in the determination of the hydraulic behavior of reservoirs proposing an analytical geomechanical model for BD's prediction in sandstone reservoirs. In practical terms, the objective is to predict whether the conditions a given portion of a siliciclastic hydrocarbon deposit have been reached for the localization of BD or how close it is to a possible generation of this feature in order to suggest an analysis of the permeability reduction. The proposal extends to the computational implementation of the method and its application in a case study of a poorly consolidated sandstone reservoir on the brazilian continental margin, rich in BD.

The results confirmed the viability of the method by showing that the portion of the analyzed reservoir reached the mechanical conditions for the genesis of $\mathrm{BD}$ from the geological age entitled Oligocene ( 23 to $35 \mathrm{Ma})$ for the established scenarios.

\section{Introdução}

A predição da permeabilidade é uma etapa crítica no fluxograma de caracterização e de modelagem geológica de reservatórios e essencial para o desenvolvimento de projetos de produção de óleo e gás. Uma das principais incertezas no processo de modelagem envolve a determinação do comportamento hidráulico de zonas de falhas, que podem atuar como condutos ou barreiras ao fluxo de fluidos no espaço e no tempo [Rotevatn e Fossen (2011), Tueckmantel et al. (2012)].

Alguns fatores contribuem para a dificuldade na caracterização dos reservatórios. Entre eles, destacamse: a complexa e heterogênea área influenciada por falhas e por estruturas subsísmicas (bandas de deformação e fraturas); a tecnologia utilizada para simulação de fluxo que, por sua complexidade computacional, ainda utiliza malhas grossas com células em torno de 50 metros de espessura, resultando em representações pobres das falhas; e a dificuldade e custo na obtenção de amostras de rochas em águas profundas para a descrição de suas propriedades mecânicas e petrofísicas.

Por serem subsísmicas, as bandas de deformação (BD) são feições de difícil mapeamento, porém, são comuns em reservatórios siliciclásticos com alta porosidade e podem reduzir consideravelmente a permeabilidade do sistema em relação à permeabilidade da rocha original.

O presente trabalho propõe a elaboração e implementação computacional de um modelo geomecânico analítico para a predição de $\mathrm{BD}$ em reservatórios de arenitos porosos. A ferramenta desenvolvida é capaz de auxiliar os profissionais de geo-engenharia em análises de sensibilidade para estimarem qualitativamente o potencial de formação de BD e seus efeitos na qualidade dos reservatórios.

O modelo proposto fundamenta-se nos conhecimentos adquiridos nos últimos anos sobre o impacto das BD na redução de permeabilidade em arenitos, nos fatores que controlam a intensidade de catáclase, na mecânica de geração dessas estruturas, e na teoria do estado crítico da mecânica dos solos, amplamente utilizada no entendimento do desenvolvimento de BD [Ballas et al. (2015), Fossen et al. (2017)]. Tal abordagem baseia-se também no estudo da geo-história da unidade estratigráfica em questão, com a finalidade de se obter os paleomodelos geomecânicos, indispensáveis para traçar a trajetória de tensões de um ponto ou determinada porção do reservatório desde a deposição até a posição atual.

A proposta se estende na aplicação do método via estudo de caso de um reservatório arenítico de petróleo de uma bacia sedimentar da Margem Continental Brasileira.

\section{Método}

As BD são estruturas subsísmicas de deformação, localizadas em uma dimensão finita de rocha, comuns em arenitos porosos e que variam em relação a mecanismos de deformação, geometria e distribuição. Exemplos de afloramentos com BD podem ser visualizados na Figura 1.

A quantidade de catáclase envolvida na deformação determina, em grande parte, como as BD afetam o fluxo de fluido. Catáclase pode ser definida como uma deformação tectônica acompanhada da trituração ou cominuição generalizada dos grãos. Muitas vezes pode originar até outro tipo de rocha, como o gouge de falha. Normalmente, quanto mais intenso for esse processo uma maior redução de permeabilidade se verifica. A cominuição dos grãos é geralmente facilitada por grãos grossos e bem selecionados, por alta porosidade (geralmente maior que $15 \%$ ) e por soterramentos elevados (geralmente maiores que 500 a $1000 \mathrm{~m}$ ) no momento da plastificação. 


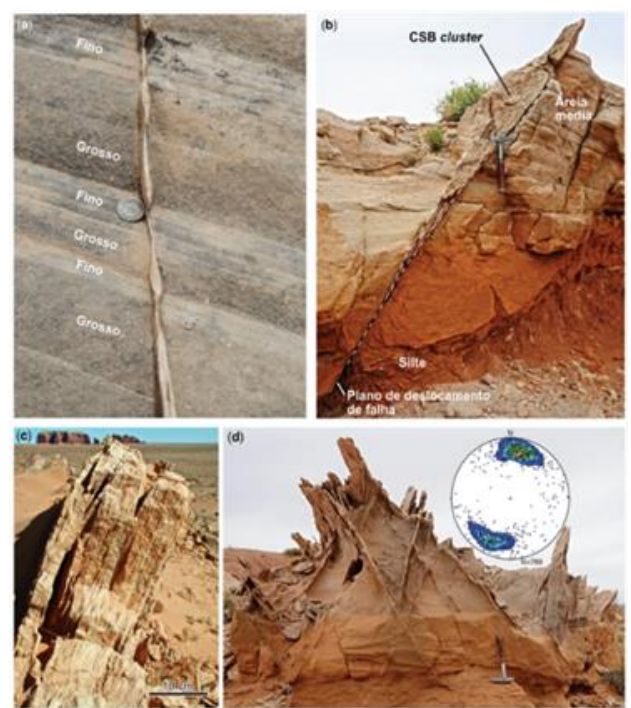

Figura 1 - Exemplos de bandas de deformação. Adaptado de Fossen et al., 2017.

Além do processo de catáclase, o desenvolvimento das $\mathrm{BD}$ envolve outros mecanismos em microescala como o esmagamento, fraturamento, atrito, rotação e deslizamento dos grãos, além de dissolução e cimentação (Fossen et al., 2017).

Muitos estudiosos classificaram as BD sob diferentes critérios: intensidade de catáclase, fluxo granular, presença de membrana de filossilicatos, dissolução e cimentação, deslocamento e pela cinemática (Figura 2) (e.g. Aydin, 1978; Antonellini et al., 1994; Fossen et al., 2007; Ballas et al., 2015 e Fossen et al., 2017).

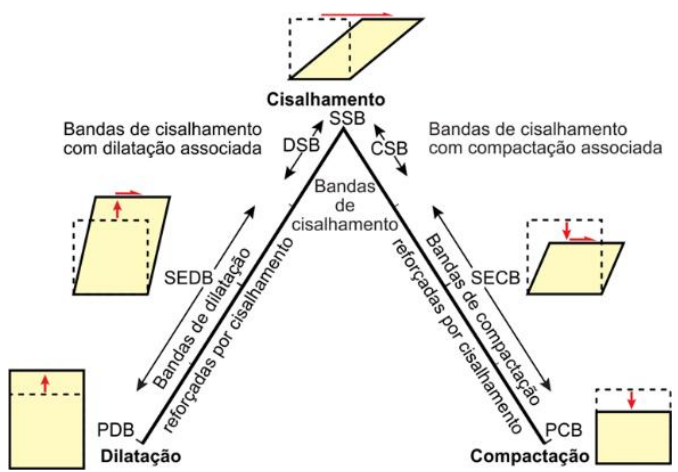

Figura 2 - Espectro cinemático de $B D$ entre os endmembers compactação, cisalhamento e dilatação. [Adaptado de Fossen et al. (2017)].

O objetivo do trabalho é prever se em uma determinada porção de um reservatório siliciclástico de hidrocarboneto foram atingidas condições para a formação de BD ou quão próximo se está de uma possível geração dessa feição a fim de se sugerir uma análise da redução de permeabilidade.

Em termos práticos, objetiva-se traçar a trajetória de tensões atuantes em uma porção do reservatório durante a formação e história de soterramento da bacia sedimentar na qual ele pertence. Para isso, é necessário: (i) o modelo geomecânico 1D atual a partir de dados do poço na profundidade alvo do estudo; (ii) as paleotensões pela geo-história, por backstripping, e pela inversão de descontinuidades, metodologia apresentada por Moraes (2018); (iii) as propriedades petrofísicas e mecânicas da rocha reservatório ao longo do tempo; (iv) a implementação de um simulador analítico com a envoltória do Modified Cam Clay Model (MCCM) e o caminho das tensões no tempo geológico.

O modelo proposto é representado pela Figura 3.

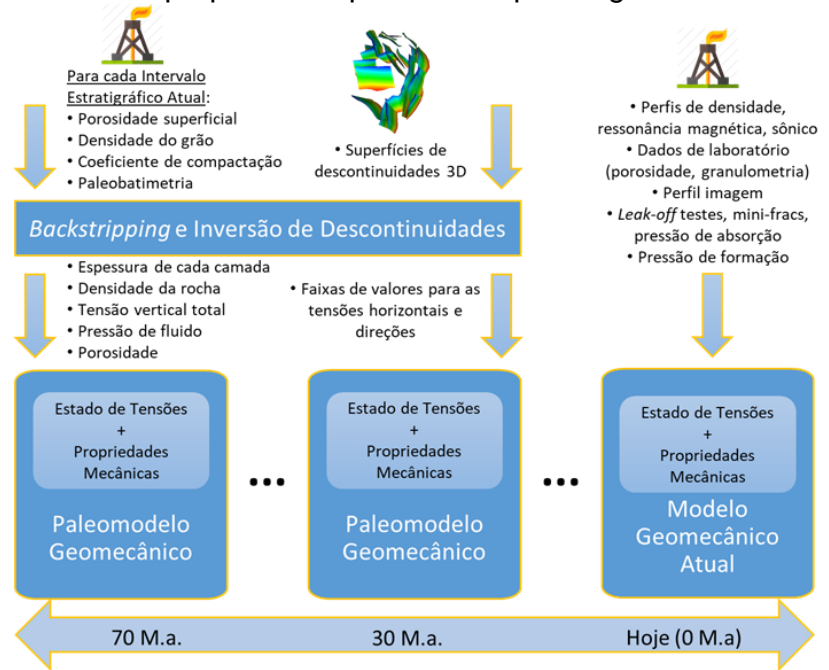

Figura 3 - Representação conceitual da metodologia.

\section{Mecânica da gênese de bandas de deformação}

Uma abordagem largamente aplicada para 0 entendimento da nucleação de BD em rocha porosa é a teoria do estado crítico, que fornece uma estrutura para descrever o comportamento inelástico de compactação e dilatância. Essa teoria é representada, no diagrama $p \times q$ (tensão média $x$ tensão diferencial), por uma envoltória limite de plastificação composta pela linha de estado crítico (CSL) e um cap (semi-elipse). A CSL separa os regimes de compressão e dilatância na superfície de plastificação (Figura 4) [Borja, 2013].

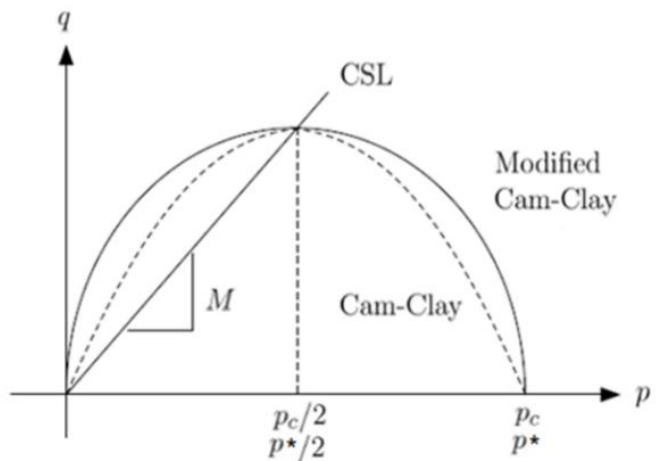

Figura 4 - Critérios de plastificação Cam Clay e Cam Clay Modificado no espaço p x q. [Adaptado de Borja (2013)].

Os modelos tipo cap são derivados da aplicação dos conceitos da mecânica dos solos aos processos de deformação inelástica observados em rochas porosas [Schultz e Siddharthan (2005)]. O trabalho original para fluxo em solos ricos em argila foi proposto por Roscoe e 
Schofield (1963) e nomeado Cam Clay Model (CCM) devido ao local donde foi retirado o material para os experimentos - Rio Cam, em Cambridge, Inglaterra. Posteriormente, foi modificado por Roscoe e Burland (1968), evoluindo para o Modified Cam Clay Model (MCCM) e se tornando o modelo do estado crítico mais amplamente utilizado.

Uma informação indispensável na modelagem da gênese de uma BD é a pressão de colapso $\left(P^{*}\right)$, pressão efetiva na qual ocorre compactação com ausência de cisalhamento $(q=0)$. Essa pressão crítica estabelece 0 limite elástico máximo para a tensão média efetiva em uma envoltória tipo cap. Fisicamente, representa o início de colapso do meio poroso com o esmagamento e o fraturamento em escala de grão.

Zhang et al. (1990b) mostraram que, para compactação hidrostática, existe um estado de tensão crítico correspondente à pressão de colapso $\left(P^{*}\right)$ que é controlado principalmente pela porosidade e pelo tamanho dos grãos. Portanto, este valor poderia ser dimensionado aproximadamente com o produto do tamanho do grão $\mathrm{R}$ pela porosidade $\Phi$, ou seja:

$$
P^{*} \propto(\Phi R)^{-1.5}
$$

Essa relação empírica, proveniente de um ajuste por regressão não linear, pode ser melhor entendida pelo diagrama mostrado na Figura 5.

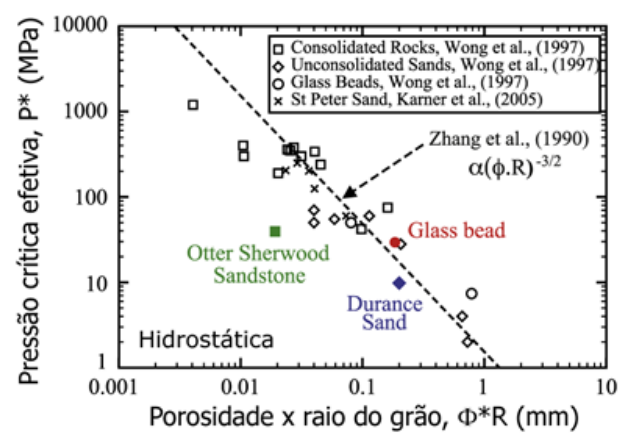

Figura 5 - Pressões de colapso do grão $\left(P^{*}\right)$ reportados por Wong et al. (1997) somados a três arenitos distintos. [Adaptado de Nguyen et al. (2014)].

Uma compilação dos dados de arenitos inconsolidados (Tabela 1) dos artigos de Wong et al. (1997) e Nguyen et al. (2014) é usada nessa modelagem para inferir o fator de proporcionalidade da equação 1 .

Tabela 1 - Compilação de dados experimentais de pressão crítica efetiva sob carga hidrostática $\left(P^{*}\right)$ de Wong et al. (1997) e Nguyen et al. (2014).

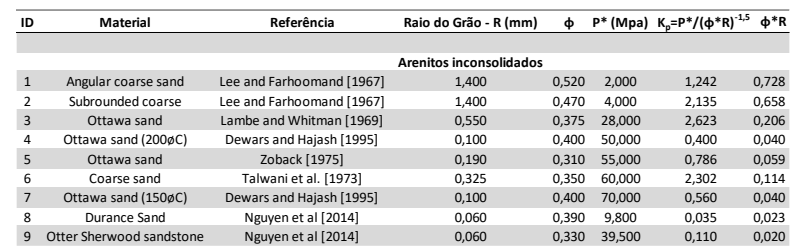

Paleotensões por Inversão de Descontinuidades

A observação de algumas propriedades cinemáticas de descontinuidades como azimute, mergulho e estrias de planos de falhas fornece insumos para a interpretação e análise de paleotensões. Quando se tem dados de um conjunto de descontinuidades, é possível estimar a orientação das tensões principais $\left(\sigma_{1}, \sigma_{2}\right.$ e $\left.\sigma_{3}\right)$. O método de paleotensões se apoia em algumas premissas: um agrupamento de falhas é formado pelo mesmo estado de tensões, as rochas são razoavelmente homogêneas, as deformações são relativamente baixas e as estruturas não sofreram rotações significantes desde sua gênese [Fossen (2011)].

Uma outra suposição bastante conhecida e essencial para a inversão de descontinuidades é a hipótese de WallaceBott [sintetizada de Wallace (1951) e Bott (1959)]. Esta afirma que a direção de deslizamento em uma descontinuidade cisalhante é paralela à maior tensão cisalhante que se dispôs na superfície da falha. Nesse sentido, deve-se minimizar o ângulo de desvio entre a direção de deslizamento real e a teórica pelo produto escalar

$$
s \cdot \sigma_{c}^{u} \leq \varepsilon+1
$$

onde s é o vetor unitário na direção da estria, $\sigma_{c}^{u}$ é o vetor unitário na direção da maior tensão cisalhante projetada na falha e $\varepsilon$ é o erro de aceitação.

\section{Backstripping}

A mecânica da geração de $\mathrm{BD}$ remete ao conhecimento do passado geológico para modelar a trajetória das tensões que atuaram na rocha reservatório. Para isso, são necessárias informações pretéritas dos estados de tensões e propriedades das rochas, ou seja, o paleomodelo geomecânico [Moraes (2018)].

De uma forma sintética o método de Backstripping tratase da descompactação sequencial das camadas estratigráficas atuais prevendo como elas estariam em diferentes tempos no passado, separando a influência do carregamento dos sedimentos sobrejacentes e a influência da tectônica na subsidência do embasamento (Allen e Allen, 2005).

Essa técnica é utilizada no modelo proposto para estimar a tensão vertical total, a pressão de poros hidrostática e a porosidade com suas derivações em diferentes tempos geológicos, permitindo a aplicação da teoria do estado crítico pelo modelo de deformação inelástica MCCM, inferindo-se o potencial de nucleação de BD.

\section{Estudo de Caso}

Muitos reservatórios descobertos no offshore do Mar do Norte, Golfo do México, Brasil e Angola são compostos por arenitos pouco consolidados que são mais propensos à compactação [Nguyen et al. (2014)]. Levando-se em conta essa informação, escolheu-se então, para uma prova de conceito, um reservatório arenítico pouco consolidado da margem continental brasileira. Para o estudo foram utilizados os dados de perfuração e de análises de laboratório de um dos poços produtores, denominado poço A. Esse poço é conhecido por apresentar feições interpretadas como BD em testemunhos e perfil imagem (Figura 6).

Para a validação do modelo de predição de BD foi selecionada a profundidade de $3.355,00$ metros. 


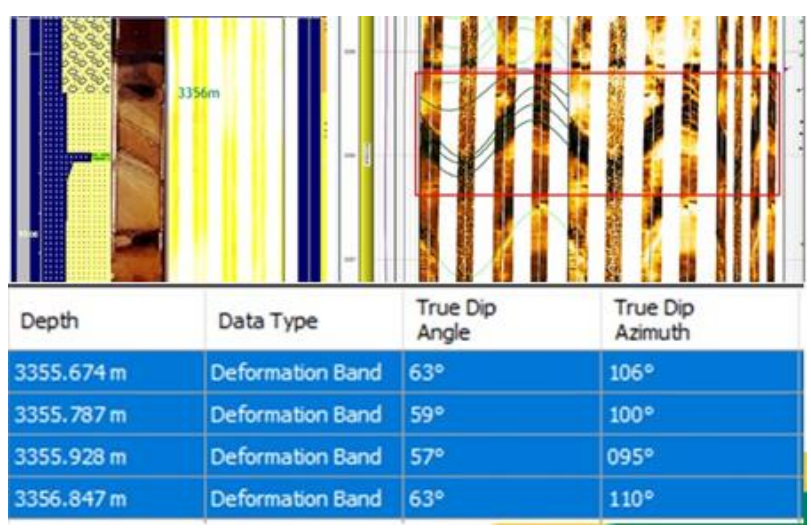

Figura 6 - Interpretação de BD em perfil imagem do poço A e suas atitudes.

O modelo geomecânico atual foi elaborado a partir do fluxo de trabalho amplamente utilizado na indústria do petróleo usando o software Sigeo3. A tensão vertical total foi obtida pelo somatório dos pesos das camadas sobrepostas ao elemento de rocha na profundidade alvo, incluindo a lâmina d'água. A tensão horizontal mínima foi estimada a partir de dados de teste de Absorção (LOT) realizado no poço A. O ângulo de abertura de quatro breakouts interpretados de perfil imagem foram utilizados para o cálculo da tensão horizontal máxima pelo método do Zoback (2010), que por sua vez foi validada pelo Polígono de Tensões. Adotou-se pressão de poros hidrostática e as propriedades físicas ângulo de atrito interno $(\varphi)$ e UCS foram estimadas a partir de correlações empíricas por litologia. Um resumo do modelo geomecânico 1D na profundidade $3.355,00 \mathrm{~m}$ é descrito na Tabela 2. Pode-se observar nesse modelo que as magnitudes das tensões totais são similares, se aproximando de um regime hidrostático.

Tabela 2 - Modelo geomecânico 1D atual na profundidade $3.355,22 \mathrm{~m}$.

\begin{tabular}{ccccccccc}
\hline PROF. (m) & $\sigma_{\mathrm{v}}(\mathrm{MPa})$ & $\mathrm{P}_{\mathrm{P}}(\mathrm{MPa})$ & peso lama (MPa) & $\sigma \mathrm{oh}(\mathrm{MPa})$ & $\sigma \mathrm{H}(\mathrm{MPa})$ & $\mathrm{UCS}(\mathrm{MPa})$ & $\boldsymbol{\varphi}\left({ }^{\circ}\right)$ & $\boldsymbol{\phi}(\%)$ \\
\hline 3355,22 & 48,32 & 33,54 & 34.53 & 44,32 & 45,420 & 14,75 & 30,51 & 29
\end{tabular}

\section{Geo-história}

Com o propósito de contar o passado geológico do reservatório adotou-se uma modelagem forward 1D por Backstripping, lançando mão do conhecimento presente pela análise dos dados do poço $A$ e de correlações empíricas de análogos disponíveis na literatura. Com essa técnica obtém-se a densidade média da coluna de rocha e a porosidade da unidade estratigráfica alvo do estudo em cada tempo geológico. Com essas informações é possível estimar também a tensão vertical e a pressão de poros, tomada como hidrostática, nos diferentes tempos. A maior incerteza nesse fluxo de trabalho é a magnitude das tensões horizontais, onde o método de inversão de descontinuidades dá a sua contribuição, restringindo ou limitando os possíveis regimes de tensões, podendo também definir uma faixa de magnitudes para o ângulo de atrito interno e direção da tensão horizontal máxima.

Diante da grande incerteza relacionada ao regime de falhamento nos diferentes tempos geológicos a técnica de inversão de descontinuidades (de fraturas, de falhas) para tensões foi utilizada em algumas falhas (Figura 7), mapeadas por interpretação sísmica e localizada nas proximidades do poço $A$, com a finalidade de estabelecer ou restringir os possíveis regimes de tensões. Porém, devido à inexistência de dados de estrias foi adotada a abordagem de Moraes e Riccomini (2014), uma alternativa ao método tradicional.

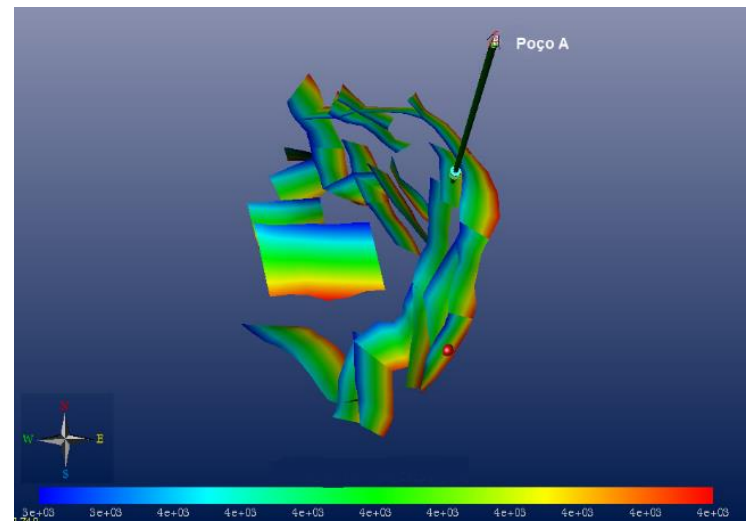

Figura 7 - Superfícies de falhas próximas ao poço A utilizadas para a inversão de descontinuidades.

A aplicação do método de inversão nas falhas mostrou que entre 30 a $60 \mathrm{Ma}$ o regime de falha era normal, as magnitudes das tensões horizontais tinham pouca diferença e o ângulo de atrito interno ficava na faixa de $12^{\circ}$ a $21^{\circ}$. O método não conseguiu uma direção única para o $\sigma_{H}$, possivelmente resultante da atuação de um estado de tensão aproximadamente isotrópico (i.e sem direção definida). De acordo com estas informações, elaborou-se alguns cenários para as tensões horizontais e regimes de falhamento por unidade estratigráfica, descritos na Tabela 3.

Tabela 3 - Cenários para as tensões horizontais e regimes de falhamento.

\begin{tabular}{|c|c|c|c|c|c|c|}
\hline \multirow{3}{*}{ Cronoestratigrafia } & \multirow{3}{*}{$\begin{array}{l}\text { Regime } \\
\text { de Falha }\end{array}$} & \multicolumn{4}{|c|}{ Cenários } & \multirow{3}{*}{ Estruturas Formadas } \\
\hline & & 1 & 2 & 3 & 4 & \\
\hline & & $K_{H} \mid K_{h}$ & $K_{H} \mid K_{h}$ & $K_{H} \mid K_{h}$ & $K_{H} \mid K_{h}$ & \\
\hline Soterramento Final & $\mathrm{n}$ & $0,94 \mid 0,91$ & $0,94 \mid 0,92$ & $0,95 \mid 0,93$ & $0,98 \mid 0,95$ & - \\
\hline Oligoceno Superior & $n$, rout & $0,8 \mid 0,8$ & $0,85 \mid 0,85$ & $1,2 \mid 0,95$ & $1,3 \mid 1,1$ & BD \\
\hline Oligoceno Médio/Inferior & $\mathrm{n}$ & $0,8 \mid 0,8$ & $0,85 \mid 0,85$ & $0,9 \mid 0,9$ & $0,95 \mid 0,95$ & falhas normais \\
\hline Eoceno & $\mathrm{n}$ & $0,8 \mid 0,8$ & $0,85 \mid 0,85$ & $0,9 \mid 0,9$ & $0,95 \mid 0,95$ & falhas normais \\
\hline Paleoceno & $\mathrm{n}$ & $0,8 \mid 0,8$ & $0,85 \mid 0,85$ & $0,9 \mid 0,9$ & $0,95 \mid 0,95$ & falhas normais \\
\hline Maastrichtiano - Res 1 & $\mathrm{n}$ & $0,8 \mid 0,8$ & $0,85 \mid 0,85$ & $0,9 \mid 0,9$ & $0,95 \mid 0,95$ & - \\
\hline Maastrichtiano - Res 2 & $\mathrm{n}$ & $0,98 \mid 0,98$ & $0,99 \mid 0,99$ & $1 \mid 1$ & $1 \mid 1$ & - \\
\hline
\end{tabular}

Inferiu-se a magnitude da pressão de colapso pela eq. 1, dependente da porosidade e do raio do grão, o que torna viável a estimativa também para os tempos pretéritos. Como essa relação exige um fator de proporcionalidade $\left(k_{p}\right)$, aplicou-se primeiramente uma regressão linear em todos os dados da Tabela 1. Porém, a reta de tendência não obteve uma boa correlação com os dados $\left(\mathrm{R}^{2}=\right.$ 0,2281 ). Sendo assim, experimentou-se outras duas regressões distintas, uma sem os arenitos 3 e 6 da Tabela 1 (Figura 8A), que resultou na relação $K_{p}=1,9647$ * $(\Phi R)+0,3125$, com valores resultantes variando entre 0,384 e 1,191; e uma outra regressão, sem os arenitos 1 e 2 da Tabela 1 (Figura 8B), que resultou na relação $K_{p}=$ $14,834 *(\Phi R)-0,0903$, com magnitudes resultantes oscilando entre 0,447 e 6,54 . 


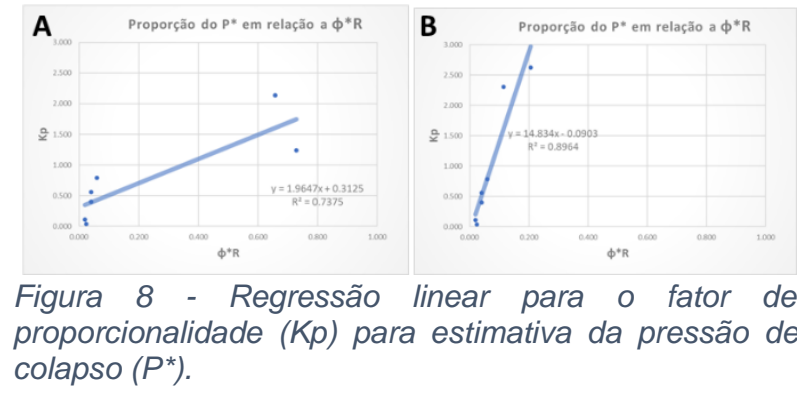

\section{Resultados}

Para a simulação definiu-se cenários com a variação dos parâmetros $K_{p}, K_{H}, K_{h}$ e $R$ (raio do grão). Como o fator $K_{p}=14,834 *(\Phi R)-0,0903$ apresentou o valor máximo $(6,54)$ bem acima do valor máximo de $K_{p}$ da Tabela 1 $(2,623)$ e resultou em magnitudes de $P^{*}$ na faixa entre $21,89 \mathrm{MPa}$ e $64,83 \mathrm{MPa}$, o que não é suficiente para a localização de uma BD com algum componente de compactação, decidiu-se mostrar somente os resultados referentes ao fator de proporcionalidade $K_{p}=1,9647$ * $(\Phi R)+0,3125$, que possibilita a nucleação de $\mathrm{BD}$.

As superfícies de cap formadas a partir dos raios do grão $0,250 \mathrm{~mm}$ e $0,125 \mathrm{~mm}$ também ficaram distantes dos estados de tensões nos diferentes tempos geológicos. Como se sabe que há $\mathrm{BD}$, possivelmente os tamanhos dos raios de hoje em dia não correspondem necessariamente ao passado supondo que a eq. (1) esteja de fato correta. Para uma melhor visualização dos resultados, decidiu-se eliminar esses cenários dos resultados finais.

Após a simulação (Figura 9), pode-se listar algumas considerações:

(i) na primeira fase do Maastrichtiano ( 70 Ma), devido, talvez, à extensa lâmina d'água $(1.610 \mathrm{~m})$, a magnitude da $P_{p}(16,91 \mathrm{MPa})$ se aproxima da $\sigma_{v}$ $(17,83 \mathrm{MPa})$ resultando em um regime praticamente hidrostático;

(ii) na segunda fase do Maastrichtiano ( $\sim 5 \mathrm{Ma})$ quanto maior a tensão diferencial, mais o estado de tensões se aproxima ou ultrapassa o limite para plastificação por dilatação, conforme o esquema conceitual de Schultz e Siddharthan (2005);

(iii) no Paleoceno ( $60 \mathrm{Ma}$ ) percebe-se uma tendência de diminuição da tensão diferencial e aumento da tensão média, o que torna a porção do reservatório mecanicamente mais estável, i.e., mais distante de uma deformação inelástica, com exceção do cenário $K_{H}=K_{h}=0,8$ que se encontra acima da CSL;

(iv) no Eoceno ( 50 Ma) vê-se uma aproximação maior do cap para grãos mais grossos $(R=1 \mathrm{~mm})$, podendo-se até afirmar que se a $P_{p}$ fosse um pouco menor seria possível a nucleação de uma BD com um forte componente de compactação. Porém, no cenário $K_{H}$ $=K_{h}=0,8$ o estado de tensões continua acima da CSL;

(v) no Oligoceno Médio/Inferior ( 35 Ma) observa-se o mesmo comportamento da idade anterior. Entretanto, para o cenário $\left(K_{H}=0,8, K_{h}=0,8\right)$ o estado de tensões ultrapassa o limite de plastificação dúctil para o cap de $\mathrm{R}=1 \mathrm{~mm}$, o que caracterizaria uma CSB (banda de cisalhamento com compactação associada);

(vi) no Oligoceno Superior ( 25 Ma) vê-se uma alta probabilidade da localização das BD vistas no testemunho do poço A (Figura 6) na profundidade aproximada de $3.355,00 \mathrm{~m}$. Em todos os cenários o caminho das tensões resultam em deformações hardening para o cap de $\mathrm{R}=1 \mathrm{~mm}$, sendo que nos cenários de regimes transcorrente e reverso a trajetória de tensões também ultrapassa o cap de $R=$ $0,5 \mathrm{~mm}$;

(vii) no tempo atual $(0 \mathrm{Ma})$ tem-se a situação mais provável para a nucleação de BD com forte grau de compactação para ambos os caps $(R=1 \mathrm{~mm}$ e $R=$ $0,5 \mathrm{~mm}$ ).

\section{Conclusões}

Os resultados confirmaram a aplicabilidade prática da metodologia. De acordo com o que foi postulado, observase uma probabilidade crescente da formação das BD visualizadas em testemunho do Poço A a partir do Oligoceno ( 23 a $35 \mathrm{Ma}$ ). Nesse período, as feições seriam localizadas nos cenários de regime de falhamento transcorrente e reverso em grãos de raios $1 \mathrm{~mm}$ e $0,5 \mathrm{~mm}$ e em cenários de regime de falha normal em grãos de raio $1 \mathrm{~mm}$. Um regime transcorrente ou reverso aumentaria o potencial da nucleação de BD nessa idade geológica. O tempo presente reafirma as condições para a gênese dessas estruturas, porém, em regime normal.

A maior dificuldade na modelagem se deu na definição da pressão efetiva crítica de colapso $\left(P^{*}\right)$. Na falta de ensaios hidrostáticos, a relação de Zhang et al. (1990b) possibilitou a inferência da $P^{*}$ nos diferentes tempos geológicos via porosidade e raio do grão. Contudo, observou-se que essa relação empírica não encerra a magnitude de $P^{*}$, pois há uma grande variação no fator de proporcionalidade devido as diferenças composicionais dos arenitos e da existência restrita de resultados de ensaios hidrostáticos na literatura.

\section{Agradecimentos}

À Petrobras e à PUC-Rio por apoiarem essa pesquisa.

\section{Referências}

Allen, P.A.; Allen, J.R. Basin Analysis: Principles and Applications. $2^{\mathrm{a}}$ ed., Blackwell Publishing (2005), p. 351, 359-366.

Antonellini, M.A.; Aydin, A.; Pollard, D.D. Microstructure of deformation bands in porous sandstones at Arches National Park, Utah. Journal of Structural Geology, 16 (1994) 941-959, 1994.

Aydin, A. Small faults formed as deformation bands in sandstones. Pure and Applied Geophysics (1978), 116: 913-930.

Ballas, G.; Fossen, H.; Soliva, R. Factors controlling permeability of cataclastic deformation bands and faults in porous sandstone reservoirs. Journal of Structural Geology 76 (2015) 1-21.

Borja, R.I. Plasticity Modeling \& Computation. $1^{\frac{a}{}}$ ed., Springer (2013), p. 122. 

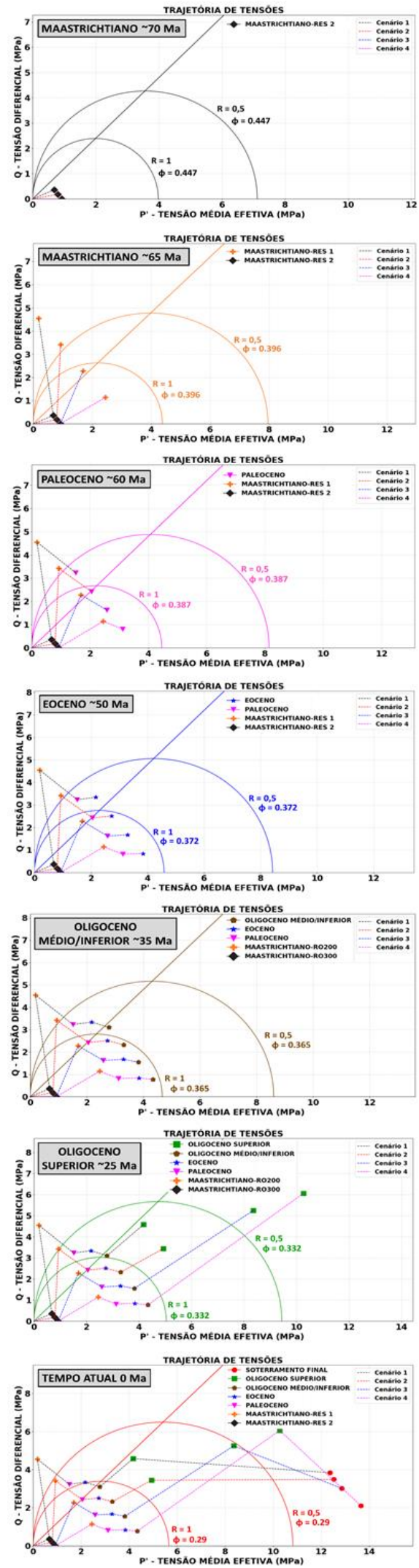

Figura 9 - Resultados das simulações.
Bott, M. The mechanics of oblique slip faulting. Geological Magazine (1959), 96:109-117.

Fossen, H.; Schultz, R.A.; Shipton, Z.K.; Mair, K. Deformation bands in sandstone: a review. Journal of the Geological Society (2007) 164 (4), 755-769.

Fossen, H.; Soliva, R.; Ballas, G.; Trzaskos, B.; Cavalcante, C.; Schultz, R.A. A review of deformation bands in reservoir sandstones: geometries, mechanisms and distribution. Geological Society, London, Special Publications, 459 (2017) 9-33.

Fossen H. Structural Geology. $2^{\mathrm{a}}$ ed., Cambridge University Press (2011), p. 192-196.

Moraes, A.; Riccomini, C. Inversão de paleotensões em bacias sedimentares utilizando condicionantes da geomecânica aplicada à indústria do petróleo. $47^{\circ}$ Congresso Brasileiro Geologia (2014), Salvador PAP15206.

Moraes, A. Estimativa de Paleomodelos Geomecânicos em Bacias Sedimentares. I Simpósio de Geologia (2018), Petrobras.

Nguyen, V.H.; Gland, N.; Dautriat, J.; David, C.; Wassermann, J.; Guélard, J. Compaction, permeability evolution and stress path effects in unconsolidated sand and weakly consolidated sandstone. International Journal of Rock Mechanics \& Mining Sciences 67 (2014) 226-239.

Roscoe, K.H., Burland, J.B. On the generalized stressstrain behaviour of 'wet' clay. In: Engineering Plasticity. Cambridge University Press (1968), p. 535-609.

Roscoe, K.H.; Schofield, A.N.; Thurairajah, A. Yielding of clays in states wetter than critical. Géotechnique 13 (1963), 211-240.

Rotevatn, A; Fossen, H. Simulating the effect of subseismic fault tails and process zones in a siliciclastic reservoir analogue: Implications for aquifer support and trap definition. Marine and Petroleum Geology 28 (2011) 1648-1662.

Schultz, R.A.; Siddharthan, R. A general framework for the occurrence and faulting of deformation bands in porous granular rocks. Tectonophysics 411 (2005) 1-18.

Tueckmantel, C.; Fisher Q.J.; Grattoni C.A.; Aplin, A.C. Single-and two-phase fluid flow properties of cataclastic fault rocks in porous sandstone. Marine and Petroleum Geology 29 (2012) 129-142.

Wallace, R. Geometry of shearing stress and relation to faulting. The Journal of Geology (1951), 59:118-130.

Wong, T.; David, C.; Zhu, W. The transition from brittle faulting to cataclastic flow in porous sandstones: Mechanical deformation. Journal of Geophysical Research 102 (B2) (1997) 3009-3025.

Zhang, J.; Wong, T.; Davis, D.M. Micromechanics of Pressure-Induced Grain Crushing in Porous Rocks. Journal of Geophysical Research, 95 (B1) (1990) 341-352.

Zoback, M.D. Reservoir Geomechanics. $2^{\mathrm{a}}$ ed., Cambridge University Press (2010), p. 222-223. 